\title{
AVALIAÇÃO DO DESEMPENHO DE MICROBICIDAS PARA A PRESERVAÇÃO DE COUROS
}

\author{
J. T. DA FONTOURA ${ }^{1}$, D. ODY ${ }^{1}$, A. BRANDELLI ${ }^{2}$, M. GUTTERRES ${ }^{1}$ \\ ${ }^{1}$ Universidade Federal do Rio Grande do Sul (UFRGS), Departamento de Engenharia \\ Química, Laboratório de Estudos em Couro e Meio Ambiente (LACOURO) \\ ${ }^{2}$ Universidade Federal do Rio Grande do Sul (UFRGS), Instituto de Ciência e Tecnologia dos \\ Alimentos, Departamento de Ciências dos Alimentos. \\ E-mail para contato: \{jutolfo, mariliz\}@enq.ufrgs.br
}

\begin{abstract}
RESUMO - Uma das grandes preocupações na indústria do couro é a deterioração de couros devido ao desenvolvimento de fungos que causam manchas e modificações nas propriedades físico-mecânicas, afetando a qualidade do produto final. Surge assim, a necessidade de desenvolver estratégias de controle, recorrendo-se à utilização de microbicidas. Tendo em vista a melhoria deste processo, este trabalho tem por objetivo avaliar o desempenho de cinco microbicidas (TCMTB, Isotiazolina, OIT+BMC/óleo, OIT+BMC/água, OIT) comumente utilizados na indústria coureira contra diferentes fungos. Os microbicidas foram aplicados no processo de curtimento ao cromo. Para verificar a proteção antifúngica destes microbicidas foram realizados testes microbiológicos acelerados de plaqueamento e de acondicionamento em câmara tropical, além da microscopia eletrônica de varredura (MEV) para verificar os danos causados ao couro devido ao ataque dos fungos. Dois dos microbicidas estudados, TCMTB e OIT+BMC/água aplicados no wet-blue, revelaram uma elevada capacidade antifúngica contras os diferentes fungos testados como contaminantes.
\end{abstract}

Palavras-chave: microbicida, biodeterioração do couro, Testes microbiológicos acelerados

\section{INTRODUÇÃO}

O couro, cuja matéria-prima básica são as peles de animais, é um produto industrial intermediário com inúmeras aplicações em diferentes setores como calçados, vestuário, estofamentos e artefatos diversos. As peles brutas são constituídas basicamente por água, responsável por quase $60 \%$ a $70 \%$ em peso, e proteínas que são biopolímeros formados de polipeptídios, em níveis de aproximadamente 30\%. A principal proteína da pele é o colágeno, a qual representa um terço do total de proteínas dos mamíferos (Kanagaraj et al., 2005; Adzet, 2010; Joseph e Nithya, 2009).

As peles e couros, por possuírem alta umidade e serem ricas em gordura e proteína, servem como substratos metabólicos para os microrganismos, especialmente bactérias e fungos. Estes microrganismos usam os nutrientes presentes nas peles como fonte de alimentação e produção de energia e são capazes de contaminar as peles desde o abate, até o couro já processado (Bryant et al., 2011; Orlita, 2004). 
Na biodeterioração de peles e couros, os microrganismos utilizam dois tipos de enzimas, as peptidases e proteinases. Essas enzimas reagem com estes hidrolisando gorduras, proteínas e carboidratos em estruturas menores, que são facilmente metabolizadas por fungos e bactérias (Arroyo, 2009; Cuadros et al., 2011).

Em peles brutas (ou verdes) se a contaminação microbiana não for contida, ou mesmo durante $\mathrm{o}$ processamento nas etapas de ribeira e curtimento, o produto final terá consequências prejudiciais à qualidade como machas pigmentadas de difícil remoção, liberação de ácidos graxos, defeitos, aspereza superficial e propriedades físico-mecânicas comprometidas devido à degradação do colágeno, que reduzem o valor do couro ou dos produtos com ele fabricado. Não obstante as perdas financeiras há também as perdas indiretas na forma de retrabalho (custo de mão de obra e produtos químicos) e insatisfação dos clientes (entregas atrasadas) além de ser um perigo potencial para a saúde devido à produção de micotoxinas (Haibin et al., 2011; Orlita, 2004; Stockman et al., 2006).

Desta forma, surge a necessidade de desenvolver estratégias de controle dos microrganismos de modo a reduzir ou eliminar este problema. Para tanto, recorre-se, comumente, à utilização de microbicidas. Microbicida pode ser definido como qualquer substância, química ou natural, que contém um ou mais agentes ativos, destinados a destruir, tornar inofensivo, evitar a ação ou exercer controle sobre qualquer microrganismo considerado nocivo ao material (Paulus, 2005).

$\mathrm{Na}$ indústria do couro os microbiocidas representam cerca de $15 \%$ dos custos dos produtos químicos para processamento do couro e são compostos basicamente por ingrediente ativo, solvente e tensoativo (Ruiz et al., 2006). A eficiência de um biocida depende de alguns fatores importantes, entre eles: ótima atividade contra fungos e bactérias; compatibilidade com o couro e outros produtos químicos utilizados no processo, eficácia sob variação de $\mathrm{pH}$, estabilidade em relação à temperatura e à solubilidade, pouca toxicidade para outras formas de vida, biodegradabilidade, segurança do ponto de vista da saúde, do manuseio e armazenamento e aceitabilidade no ponto de vista ambiental (Hauber, 2008; Cuadros, 2011; Bott, 1992).

$\mathrm{Na}$ década de 70 , os couros eram protegidos com substâncias químicas como o pentaclorofenol (PCP), que foi largamente utilizado na indústria do couro e teve em anos mais recentes o seu uso e comercialização proibidos, devido à toxicidade do seu princípio ativo e aos danos causados ao meio ambiente. Por este motivo, cresce cada vez mais a procura e o desenvolvimento por fungicidas eficazes, com o menor impacto ambiental e toxicológico (Dalton, 2012; Muralidharan, 1994).

Atualmente o mercado oferece poucas substâncias ativas, sendo, geralmente produtos sintéticos. Os fungicidas mais utilizados na indústria do couro recaem principalmente em duas famílias químicas (Font et al., 2011):

-Fenólicos: que incluem p-cloro-m-cresol (PCMC) e orto-fenilfenol (OPP); e,

-Heterocíclicos: que incluem 2-(tiocianometiltio) benzotiazole (TCMTB), noctilisotiazolinona (OIT) e 2-mercaptobenzotiazol (MBT).

O principal fungicida utilizado na indústria do couro é o TCMTB, que foi indicado como um substituto dos fungicidas à base de mercúrio e fenóis, que eram muito utilizados (Reyes et al., 2002). Algumas vantagens da aplicação do TCMTB são (Cítera et al., 2006): excelente ação biocida, alta eficiência quando se exige conservação do couro por um tempo mais longo, 
solubilidade em água, boa estabilidade em meio ácido e neutro; sistema emulsionante que assegura boa dispersão e distribuição na pele e baixa toxicidade.

\section{MATERIAIS E MÉTODOS}

Para avaliar a capacidade antifúngica de cinco fungicidas comerciais, adicionados na etapa de curtimento ao cromo, foram realizados testes microbiológicos acelerados de plaqueamento e de acondicionamento dos couros em câmara tropical, além da análise por microscopia eletrônica de varredura para verificar os danos causados devido ao ataque dos fungos.

\subsection{Materiais e aplicação de fungicidas no couro}

Os seguintes fungicidas foram testados nos ensaios:

- 2-(tiocianometiltio) benzotiazole (TCMTB),

- Isotiazolina,

- Dispersão oleosa de 2-n-octil-4-isotiazolin-3-ona+carbendazim (OIT+BMC/óleo),

- Dispersão aquosa de 2-n-octil-4-isotiazolin-3-ona+carbendazim (OIT+BMC/água),

- 2-n-octil-4-isotiazolin-3-ona (OIT).

A capacidade antimicrobiana dos fungicidas selecionados foi avaliada contra o crescimento de quatro espécies de fungos: Aspergillus niger, Aspergillus flavus, Penicillium herguei e Penicillium chrysogenum. As espécies foram repicadas em placas de Petri contendo meio de cultura de batata dextrose ágar (Oxoid) e incubadas durante sete dias a temperatura de $28{ }^{\circ} \mathrm{C}$. Uma suspensão de esporos de cada uma das estirpes foi preparada em solução aquosa e ajustada para uma concentração de $10^{5}$ esporos $/ \mathrm{mL}$. A contagem de esporos foi realizada utilizando câmara de Neubauer. No teste de plaqueamento também foi utilizado o meio de cultura batata dextrose ágar (Oxoid).

Os testes foram realizados utilizando uma pele bovina piquelada de $22 \mathrm{~kg}$, da qual foram tomados seis pedaços de cerca de $40 \mathrm{~g}$, que foram submetidos ao curtimento com cromo. Os processos foram realizados em fulões de bancada, em separado para cada um dos cinco fungicidas testados, e foi feito mais um teste controle sem adição de fungicida. As quantidades (\%) dos produtos químicos utilizados para o curtimento foram calculadas com base na massa da pele (Tabela 1).

Tabela 1 - Formulação do curtimento ao cromo

\begin{tabular}{c|c|c|c}
\hline Processo & Quantidade & Produto & Controle \\
\hline \multirow{3}{*}{ Píquel } & $200 \%$ & Água & \\
& $5 \%$ & Cloreto de sódio & \\
& $0,2 \%$ & Ácido fórmico & $30 \mathrm{~min}$ \\
& $0,2 \%$ & Ácido sulfúrico & $\mathrm{pH}<3$ \\
Curtimento & $9 \%$ & Sal de cromo Cromossal-B & $120 \mathrm{~min}$ \\
Basificação & $0,2 \% *$ & Fungicida & $180 \mathrm{~min}$ \\
& $0,5 \%$ & Óxido de magnésio & $360 \mathrm{~min}$ \\
& & $\mathrm{pH}(3,8-4,2)$ \\
\hline
\end{tabular}

*com exceção da amostra controle (0\%). 


\subsection{Teste de Plaqueamento}

Amostras de $25 \mathrm{~mm}$ x $25 \mathrm{~mm}$ do couro obtido (Tabela 1) foram submetidas em duplicata para a determinação da resistência de ataque de fungos (ASTM D4576 -08) para as quatro cepas selecionadas. Após solidificação do meio, uma gota da suspensão de esporos $\left(1 \times 10^{5}\right.$ esporos/mL), foi inoculada diretamente sobre a amostra de couro e outra gota foi inoculada no meio de cultura, a uma distância de 45 mm, como mostra a Figura 1.

As placas foram incubadas à temperatura de $28{ }^{\circ} \mathrm{C}$. A observação do crescimento dos fungos foi acompanhada semanalmente até 28 dias por inspeção visual. O parâmetro chamado "distância de inibição" (Figura 1), foi a distância máxima observada desde a borda da amostra de couro até o local na placa, onde o crescimento do microrganismo foi impedido pela atividade fungicida (CUADROS et al., 2011). Foi verificado e quantificado também por visualização o crescimento supercial (CS) do fungo na própria amostra de couro wet-blue.

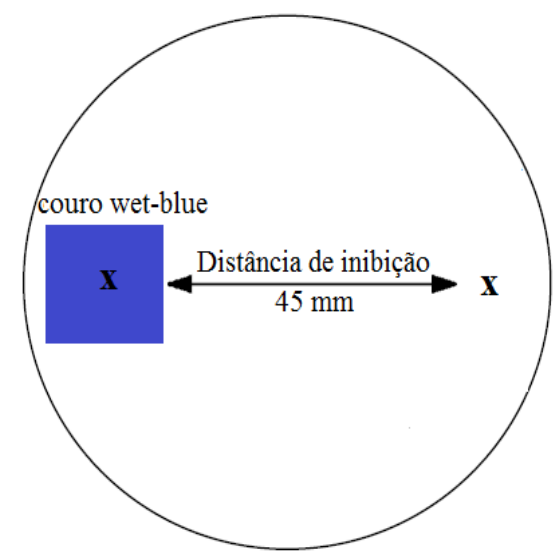

Figura 1 - Pontos de inoculação (X) da suspensão de esporos.

\subsection{Câmara tropical}

O método da câmara tropical é constituído por uma câmara construída com base na descrição da norma ASTM D7584-10, mantida a uma temperatura controlada de aproximadamente $30{ }^{\circ} \mathrm{C}$, com cerca de $100 \%$ de umidade e altamente contaminada.

Doze amostras (duplicatas dos seis testes) do couro medindo $90 \mathrm{~mm}$ x $30 \mathrm{~mm}$ sem e com fungicidas, foram colocadas na câmara tropical para avaliar o crescimento de fungos sobre a superfície. O fungo utilizado como contaminante foi o Aspergillus niger. As amostras tratadas com fungicida foram avaliadas pelo lado da flor (superfície superior) e pelo lado do carnal (superfície inferior ou subcutânea) e a contaminação foi quantificada pela percentagem de superfície atacada após 28 dias de teste.

\section{RESULTADOS E DISCUSSÃO}

$\mathrm{Na}$ Tabela 2 encontram-se os resultados médios para a distância de inibição e a porcentagem de crescimento dos fungos nas superfícies dos ensaios feitos em duplicata após 28 dias de teste. A Figura 2 mostra o crescimento dos diferentes fungos, Aspergillus niger, Aspergillus flavus, Penicillium herguei e Penicillium chrysogenum nas placas. Quanto mais próximos de $45 \mathrm{~mm}$ forem os valores da distância de inibição, maior será a proteção fornecida 
pelo microbicida, porém, uma elevada distância de inibição indica que o produto pode migrar do couro tratado para o meio, o que é uma propriedade indesejável do microbicida.

Verifica-se que a proteção antifúngica fornecida por dois dos microbicidas (TCMTB e OIT+BMC/água) foi notável, pois não foi observado o crescimento dos fungos testados nas superfícies destas amostras. Porém, o microbicida OIT+BMC/água apresentou uma distância de inibição maior que o TCMTB. Uma maior resistência do fungo Aspergillus flavus foi observada em relação a três microbicidas utilizados, Isotiazolina, OIT+BMC/óleo e OIT.

Tabela 2 - Distância de inibição (DI) e Crescimento superficial (CS) de fungos nas amostras de couro no final do teste

\begin{tabular}{c|c|c|c|c|c|c|c|c}
\hline \multirow{2}{*}{ Microbicida } & \multicolumn{2}{|c|}{$\begin{array}{c}\text { Aspergillus } \\
\text { niger }\end{array}$} & \multicolumn{2}{c|}{$\begin{array}{c}\text { Aspergillus } \\
\text { flavus }\end{array}$} & \multicolumn{2}{c|}{$\begin{array}{c}\text { Penicillium } \\
\text { herguei }\end{array}$} & \multicolumn{2}{c}{$\begin{array}{c}\text { Penicillium } \\
\text { chrysogenum }\end{array}$} \\
\cline { 2 - 8 } & DI (mm) & CS $(\%)$ & DI (mm) & CS (\%) & DI (mm) & CS (\%) & DI (mm) & CS (\%) \\
\hline Controle & 0 & 100 & 0 & 100 & 0 & 70 & 0 & 68 \\
TCMTB & 1 & 0 & 0 & 0 & 29 & 0 & 28 & 0 \\
Isotiazolina & 2 & 0 & 0 & 35 & 22 & 0 & 25 & 0 \\
OIT + BMC/óleo & 1 & 5 & 0 & 62 & 45 & 0 & 45 & 0 \\
OIT + BMC/água & 9 & 0 & 38 & 0 & 45 & 0 & 45 & 0 \\
OIT & 2 & 0 & 0 & 36 & 32 & 0 & 26 & 0 \\
\hline
\end{tabular}

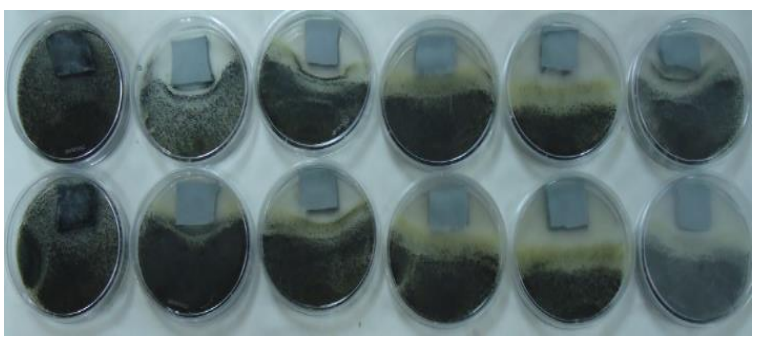

(a)

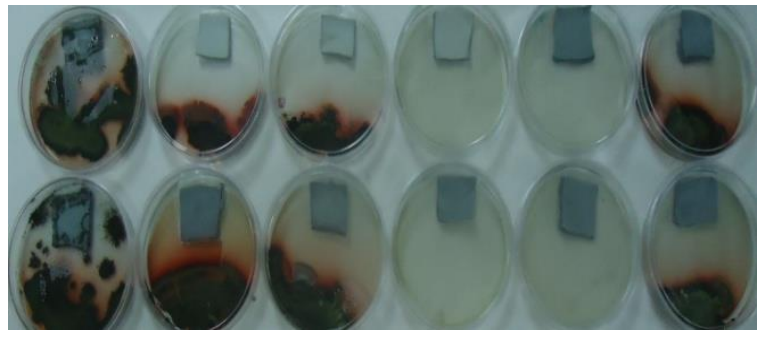

(c)

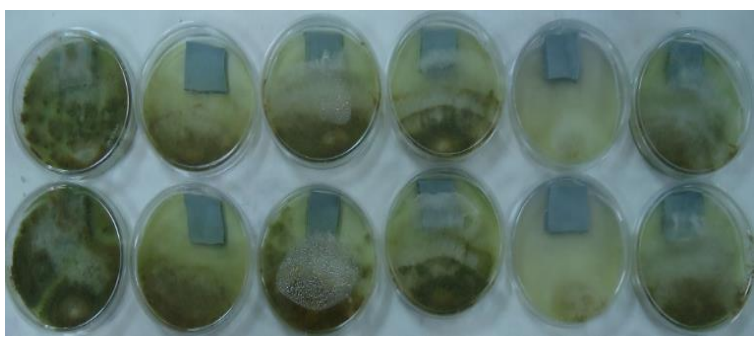

(b)

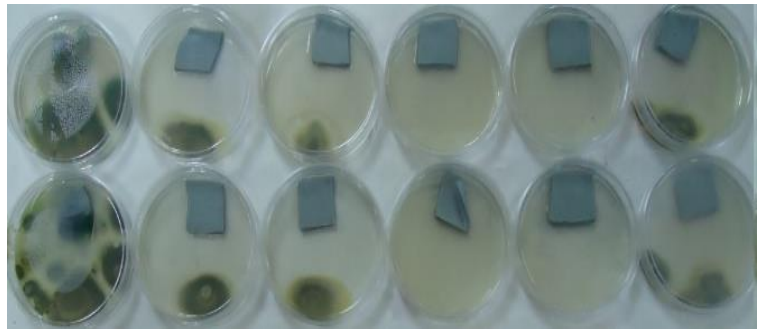

(d)

Figura 2 - Crescimento dos diferentes fungos nas amostras, da esquerda para direita: Controle,

TCMTB, Isotiazolina, OIT + BMC/óleo, OIT+BMC/água e OIT para os contaminates (a) Aspergillus niger, (b) Aspergillus flavus, (c) Penicillium herguei e (d) Penicillium chrysogenum.

As Figuras 3, 4, 5, 6 mostram a diminuição da distância de inibição para o teste de plaqueamento. A não existência de barras nos gráficos destas figuras significa que a distância entre a amostra e o crescimento do fungo foi zero. Maiores distâncias de inibição foram verificadas para Penicillium chrysogenum. Para os quatro fungos as distâncias de inibição se estabilizaram após 21 dias de teste para todos microbicidas. 


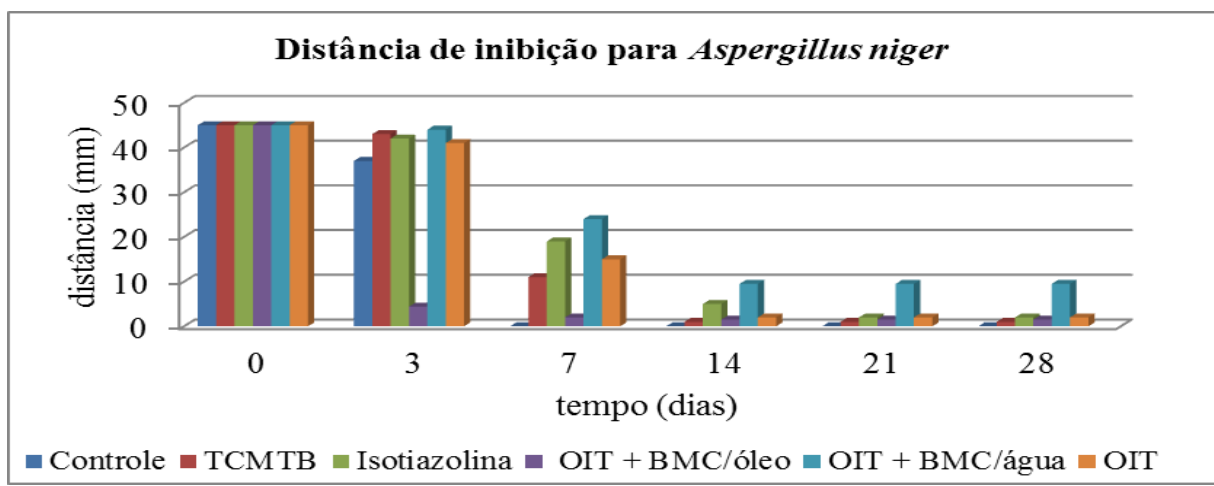

Figura 3 - Diminuição da distância de inibição com o tempo no couro contaminado com Aspergillus niger.

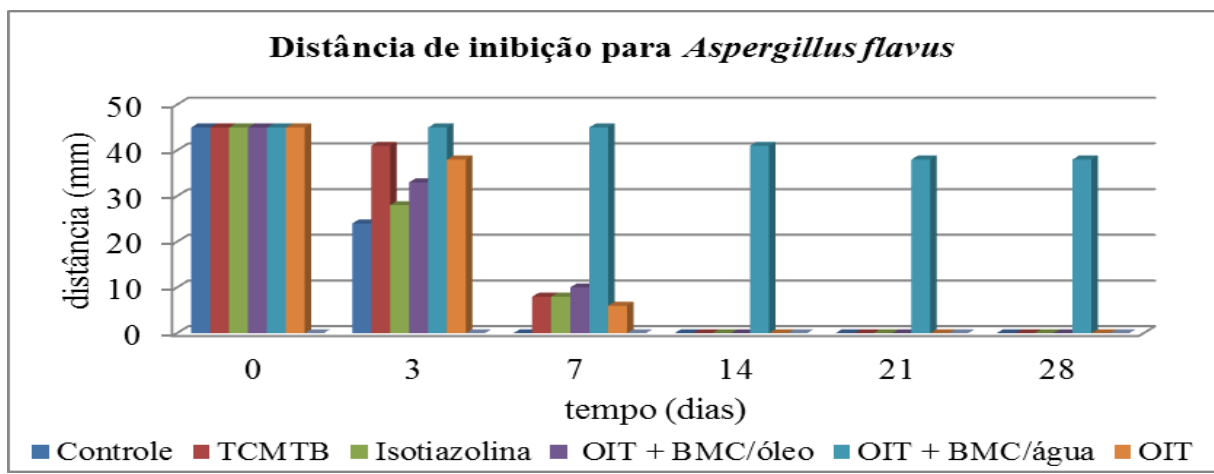

Figura 4 - Diminuição da distância de inibição com o tempo no couro contaminado com Aspergillus flavus.

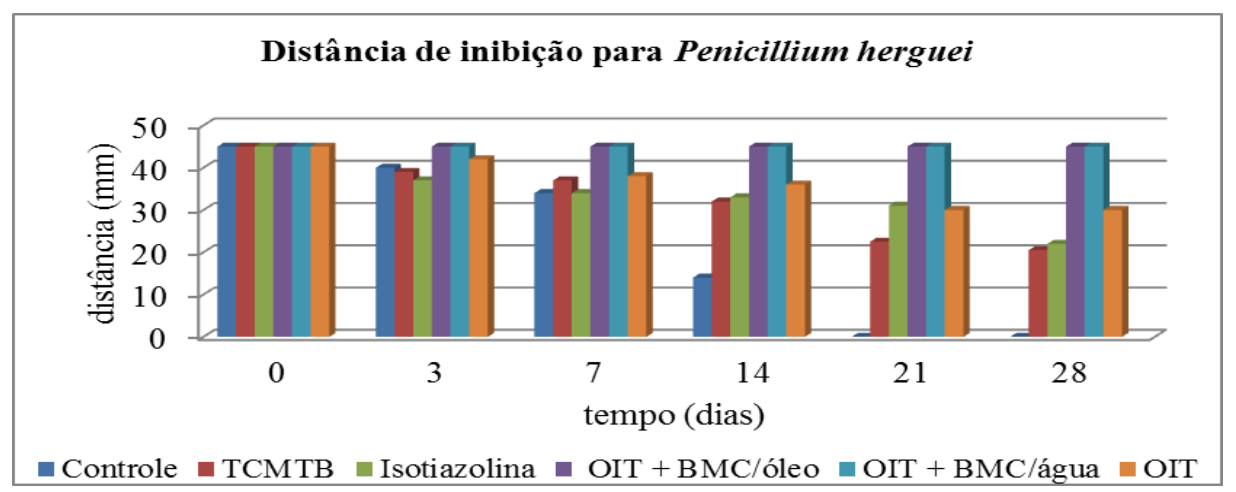

Figura 5 - Diminuição da distância de inibição com o tempo no couro contaminado com Penicillium herguei.

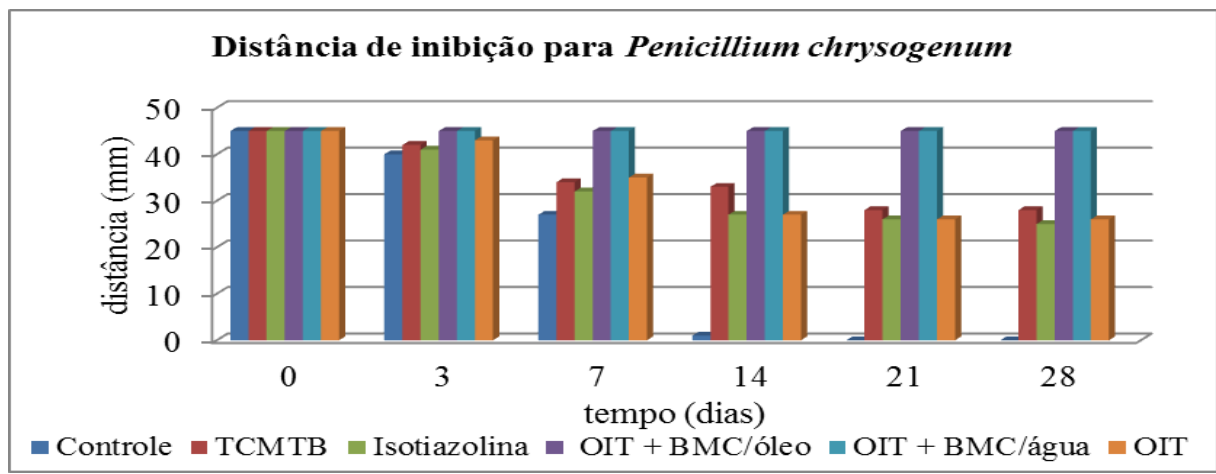

Figura 6 - Diminuição da distância de inibição com o tempo no couro contaminado com Penicillium chrysogenum. 
De acordo com as imagens, apresentadas na Figura 7, verifica-se que a amostra não contaminada ( 7 a) possui a superfícies lisa, intacta e com os poros bem definidos. Enquanto que as amostras contaminadas ( $7 \mathrm{~b}, \mathrm{c}, \mathrm{d})$ apresentam danos na superfície, pois os microrganismos ao se utilizarem do material (pele, couro), atacado para suas necessidades nutricionais, consumiram parte dele expondo as fibras, danificando-as e causando rugosidade na superfície.

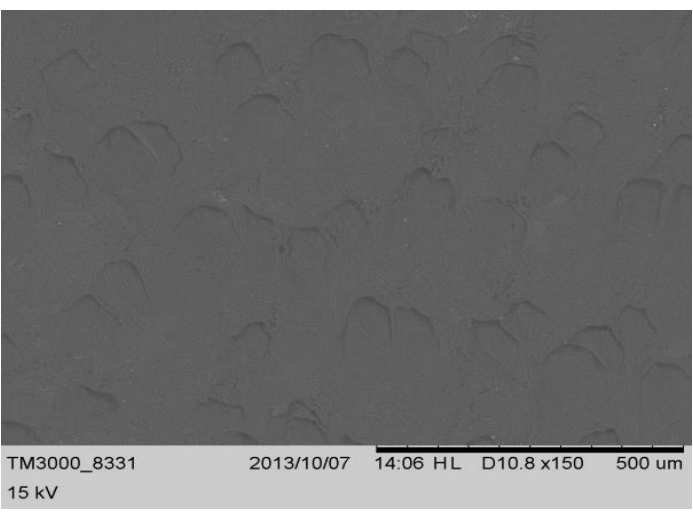

(a)

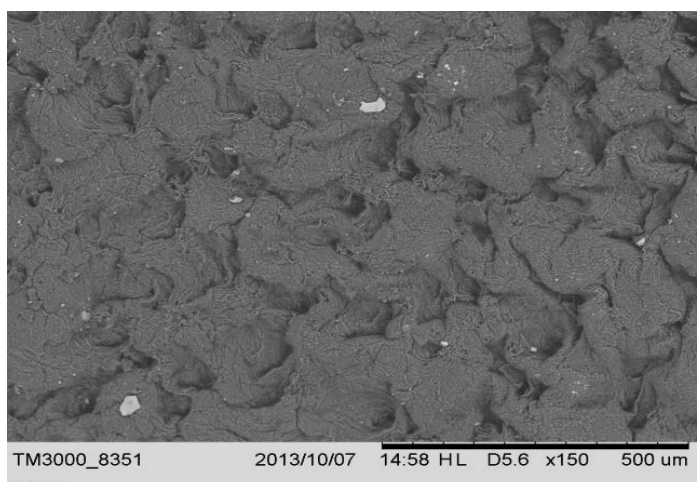

(c)

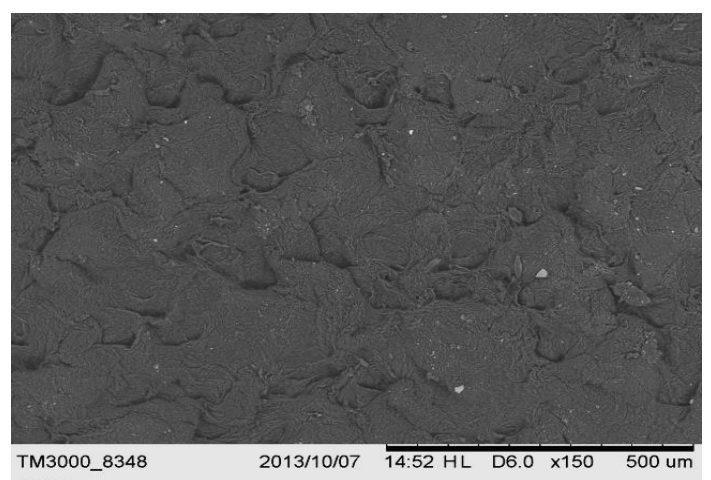

(b)

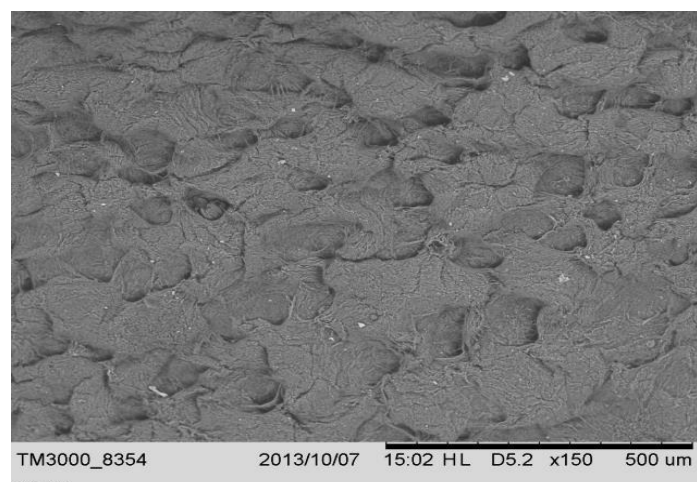

(d)

Figura 7 - Imagens das amostras de couros (a) não contaminada, (b) contaminada com Aspergillus niger, (c) contaminada com Aspergillus flavus e (d) contaminada com Penicillium herguei.

\section{CONCLUSÃO}

A partir da avaliação dos resultados obtidos e discutidos neste trabalho, pode-se concluir que o uso apropriado do microbicida é o primeiro requisito para a proteção de couros, sendo de fundamental importância para a indústria coureira.

Entre os cinco microbicidas estudados neste trabalho somente dois deles, TCMTB e OIT+BMC/água, revelaram uma elevada capacidade antifúngica contras os diferentes fungos testados. Já o OIT+BMC/óleo, na concentração indicada, não mostrou uma proteção fúngica satisfatória contra o desenvolvimento dos fungos Aspergillus niger e Aspergillus flavus, pois os mesmos mostraram-se resistentes a este microbicida.

Os resultados obtidos no teste da câmara tropical, utilizando Aspergillus niger como contaminante, mostraram compatibilidade com o teste de plaqueamento. 


\section{AGRADECIMENTOS}

Os autores agradecem à FAPERGS (Edital FAPERGS 04/2012 - Programa PqG) e ao CNPq (Edital UNIVERSAL - MCTI/CNPq No 14/2013) pelo apoio financeiro, à CAPES pela concessão de bolsa de pesquisa, à empresa Buckman Latin America e outras pelo fornecimento de parte do material utilizado para realização deste trabalho.

\section{REFERÊNCIAS BIBLIOGRÁFICAS}

ADZET, J. M. Collagen Materialis. J.A.L.C. A., v. 105, p. 254-259, 2010.

ARROYO, I.The Role of Fungi in the Deterioration of Movable and Immovable Cultural Heritage, $e$ conservation magazine v. 9, p. 40-50, 2009.

BOTT, T. R. The use of biocides in industry. In: Biofilms- Science and Technology. Applied sciences, v. 223, p. 567-581, 1992.

BRYANT, S. D.; HURLOW, E. L.; WHITTEMORE, M. S. A New Antifungal Agent for the Leather Industry: S-Hexyl-S'-Chloromethylcyanodithiocarbimate (CHED). J.S.L.T.C., v. 95, p. 07-10, 2011.

CÍTERA, G.; MARGOSIAN, G. A conservação do couro. Revista do couro, v. 181, p. 68-70, 2006.

CUADROS, S.; MANRESA, M. A.; FONT, J.; BAUTISTA, M. E.; MALDONADO, F.; MARSAL, A. Alternative Fungicides: Comparisons with Conventional Chemicals. J.S.L.T.C., v. 95, p. 263-269, 2011.

DALTON, D. L. New generation fungicide for the leather industry. J.S.L.T.C., v. 107, p. 21-30, 2012. FONT, J.; REYES, M.; CUADROS, S.; BACARDIT, A.; MARSAL, A. Determination of TCMTB and other fungicidas in leather. J.S.L.T.C., v. 106, p. 341-348, 2011

HAIBIN, G.; ZHIYUAN, W.; YANGXIN, L.; WUYONG, C. Potential fungicidal use of essential oils extracted from traditional chinese medicinal materials. J.S.L.T.C., v. 95, p. 192-199, 2011.

HAUBER, C. "Fungicides", www.leathermag.com. Published 16 august, 2008.

JOSEPH K.; NITHYA N. Material flows in the life cycle of leather. Journal of Cleaner Production v. 17, p. 676-682, 2009.

KANAGARAJ, J.; JOHN SUNDAR, V.; MURALIDHARAN, C.; SADULLA, S. Alternatives to sodium chloride in prevention of skin protein degradation da case study. Journal of Cleaner Production. v. 13, p. 825-831, 2005.

MURALIDHARAN, D.; SUNDARA RAO, V. S. Identification of leather preservatives by gas chromatography mass spectrometry. J. Chromatogr., v. 675, p. 257-260, 1994.

ORLITA, A. Microbial biodeterioration of leather and its control: a review. International Biodeterioration \& Biodegradation, v. 53, p. 157-163, 2004.

PAULUS, W. Directory of microbicides for the protection of materials. Boston, Kluwer academic publishers dordrecht, 2005.

REYES, L. H.; WRÓBEL, K.; WRÓBEL, K. Indirect extraction-spectrophotometric determination of 2-(thiocyanomethylthiol) benzothiazole in chrome tanning liquors after its breakdown to 2mercaptobenzothiazole. Talanta, v. 56, p. 515-521, 2002.

RUIZ, X. S., IZQUIERDO, A. and KOLLING, C. Desenvolvimento de novos fungicidas a base de TCMTB. Revista do Couro, v. 184, p. 76-78, 2006.

STOCKMAN, G.; DIDATO, D. T.; RANGARAJAN, R. Alternative solutions for fungal protection of packaged wet-blue. J.S.L.T.C., v. 101, p. 461-466, 2006. 